NTERnational Electronic Journal of Algebra

VOLUME 22 (2017) 97-102

DOI: $10.24330 /$ ieja.325929

\title{
A CHARACTERIZATION OF GORENSTEIN DEDEKIND DOMAINS
}

\author{
Tao Xiong \\ Received: 23 November 2016; Revised: 28 December 2016 \\ Communicated by James Zhang
}

\begin{abstract}
In this paper, we show that a domain $R$ is a Gorenstein Dedekind domain if and only if every divisible module is Gorenstein injective; if and only if every divisible module is copure injective.
\end{abstract}

Mathematics Subject Classification (2010): 16E05, 16E10

Keywords: Gorenstein Dedekind domain, divisible module, Gorenstein injective module, copure injective module

\section{Introduction}

Throughout this paper, all rings are commutative rings with identity element and all modules are unitary. For an $R$-module $M, \operatorname{pd}_{R} M\left(\right.$ resp. $\left.\operatorname{id}_{R} M, \operatorname{resp} . \mathrm{fd}_{R} M\right)$ stands for the projective (resp. injective, resp. flat) dimension of $M$. We also use $w \cdot g l \cdot \operatorname{dim}(R)($ resp. gl.dim $(R))$ to denote the weak global (resp. global) dimension of $R$.

An $R$-module $D$ is said to be divisible if $\operatorname{Ext}_{R}^{1}(R / a R, D)=0$ for all $a \in R$; and an $R$-module $M$ is called $h$-divisible if it is an epic image of an injective $R$-module. Note that injective modules and all $h$-divisible $R$-modules are divisible.

Divisible modules and $h$-divisible modules play important roles in characterizing domains. It is well known that a domain $R$ is a Dedekind (resp. Prüfer) domain if and only if every divisible module is injective (resp. FP-injective); if and only if every $h$-divisible module is injective (resp. FP-injective).

Recall that a domain $R$ is called a Matlis domain [9] if the projective dimension of the field of quotients is at most one. It is shown [10] that a domain $R$ is a

National Science Foundation of China (Grant No.11671283) and the Research Foundation for Doctor Programme (Grant No.20125134110002) and Scientific Research Fund of Sichuan Provincial Education of China (Grant No.15ZB0030) and the cultivation foundation of the outstanding senior thesis program of Sichuan Normal University (Grant No. xiaoyanzi [2015 ] 5 hao-1) and the Scientific Research Fund of Sichuan Provincial Education of China (Grant No.14ZB0035) and the Aba Teachers College Subject (Grant No.ASB14-20) and NSFC (Tianyuan Fund for Mathematics) (Grant No. 11626162). 
Matlis domain if and only if every divisible module is $h$-divisible; if and only if every divisible module is $\mathrm{K}$-injective, where an $R$-module $A$ is called K-injective if $\operatorname{Ext}_{R}^{1}(K, A)=0$ for the field of quotients $K$ of $R$.

Recall from [11] that an $R$-module $W$ is called weak-injective if $\operatorname{Ext}_{R}^{1}(M, W)=0$ for all modules $M$ with $\operatorname{fd}_{R} M \leq 1$ and from [1] that a domain $R$ is called almost perfect (APD shortly) if all its proper homomorphic images are perfect. It is proved in [8, Corollary 6.4.8] that a domain $R$ is an APD if and only if every divisible module is weak-injective; if and only if every $h$-divisible is weak-injective.

An $R$-module $M$ is said to be Gorenstein projective (G-projective for short) [5] if there is an exact sequence of projective modules

$$
\mathbf{P}=\cdots \rightarrow P_{1} \rightarrow P_{0} \rightarrow P^{0} \rightarrow P^{1} \rightarrow \cdots
$$

such that $M \cong \operatorname{Im}\left(P_{0} \rightarrow P^{0}\right)$ and that $\operatorname{Hom}_{R}(-, Q)$ leaves the sequence $\mathbf{P}$ exact whenever $Q$ is a projective $R$-module. A Gorenstein injective $R$-module is defined dually. The Gorenstein projective, injective dimensions are defined in terms of Gorenstein projective, injective resolutions, respectively, and denoted by $\operatorname{Gpd}_{R}(-)$, $\operatorname{Gid}_{R}(-)$. In [3], Bennis and Mahdou defined the Gorenstein global dimension $\operatorname{Ggldim}(R)$ of $R$, and proved that for any ring $R$, we have

$$
\begin{aligned}
\operatorname{Ggldim}(R) & =\sup \left\{\operatorname{Gpd}_{R} M \mid M \text { is any } R \text {-module }\right\} \\
& =\sup \left\{\operatorname{Gid}_{R} M \mid M \text { is any } R \text {-module }\right\} .
\end{aligned}
$$

Recall that a ring $R$ is called Gorenstein hereditary if $\operatorname{Ggldim}(R) \leq 1$. Also, a Gorenstein hereditary domain is called a Gorenstein Dedekind domain. Naturally, we propose the following question:

Question 1.1. Let $R$ be a domain. Is it true that $R$ is a Gorenstein Dedekind domain if and only if every divisible module is Gorenstein injective; if and only if every h-divisible module is Gorenstein injective?

As in [4], Enochs and Jenda introduce the concepts of copure injective modules and strongly copure injective modules. For an $R$-module $M, M$ is called copure injective if $\operatorname{Ext}_{R}^{1}(E, M)=0$ for any injective $R$-module $E$, and $M$ is called strongly copure injective if $\operatorname{Ext}_{R}^{i}(E, M)=0$ for any injective $R$-module $E$ and for all $i \geq 1$. In the paper [4] the authors define the copure injective dimension $\operatorname{cid}_{R} M$ of an $R$-module $M$ to be the largest integer $n \geq 0$ such that $\operatorname{Ext}_{R}^{n}(E, M) \neq 0$ for some injective $R$-module $E$. Of course, if no such $n$ exists, write $\operatorname{cid}_{R}(M)=\infty$. Thus $\operatorname{cid}_{R} M=0$ if and only if $M$ is strongly copure injective. As in [4, Lemma 3.1], it is shown that for an $R$-module $M, \operatorname{cid}_{R} M \leq m$ if and only if $\operatorname{Ext}_{R}^{m+i}(E, M)=0$ for 
any injective $R$-module $E$. The copure injective dimension of a ring $R$ is defined in [7] as $c i D(R)=\sup \left\{\operatorname{cid}_{R}(M) \mid M\right.$ is an $R$-module $\}$. It is clear that all domains $R$ with $\operatorname{ci} D(R) \leq 1$ are Matlis domains.

In this paper, in terms of copure injective modules, we show that a domain $R$ with $\operatorname{ci} D(R) \leq 1$ is exactly a Gorenstein Dedekind domain, and give an affirmative answer to Question 1.1.

\section{Main result}

Lemma 2.1. Let $R$ be a ring with ciD $R) \leq 1$. Then every copure injective $R$ module $M$ is divisible. Moreover, if $R$ is a domain with ciD $(R) \leq 1$, then every divisible $R$-module is copure injective.

Proof. Let $M$ be a copure injective $R$-module. For any $a \in R$ which is neither a non-zero-divisor nor a unit, $\operatorname{fd}_{R} R / a R \leq 1$ and the sequence $0 \rightarrow a R \rightarrow R \rightarrow$ $R / a R \rightarrow 0$ is exact. By hypothesis, $\operatorname{ci} D(R) \leq 1, \mathrm{fd}_{R} R^{+} \leq \operatorname{pd}_{R} R^{+} \leq 1$. Now, let $X$ be an $R$-module. Note that $(a R)^{+} \cong R^{+}$as $R$-modules. Then we can obtain $\mathrm{fd}_{R}(R / a R)^{+} \leq 1$ from the sequence $0=\operatorname{Tor}_{3}^{R}\left(X,(a R)^{+}\right) \rightarrow \operatorname{Tor}_{2}^{R}\left(X,(R / a R)^{+}\right) \rightarrow$ $\operatorname{Tor}_{2}^{R}\left(X, R^{+}\right)=0$. Then $\operatorname{id}_{R} R / a R \leq 1$ since $R / a R$ is finitely presented. So there is an exact sequence $0 \rightarrow R / a R \rightarrow E \rightarrow C \rightarrow 0$ with $E, C$ injective. Hence $\operatorname{pd}_{R} C \leq 1$ by [7]. Then $\operatorname{Ext}_{R}^{1}(E, M) \rightarrow \operatorname{Ext}_{R}^{1}(R / a R, M) \rightarrow \operatorname{Ext}_{R}^{2}(C, M)=0$ is exact. By hypothesis, $M$ is copure injective, $\operatorname{Ext}_{R}^{1}(E, M)=0$ holds. Hence $\operatorname{Ext}_{R}^{1}(R / a R, M)=0$. Thus $M$ is divisible, as desired.

Now, assmue $R$ is a domain with $\operatorname{ci} D(R) \leq 1$. Then $R$ is a Matlis domain. Let $M$ be a divisible module. By [10, Lemma 2.4], $M$ is $h$-divisible. Since $c i D(R) \leq 1$, $M$ is copure injective.

Example 2.2. A copure injective $R$-module is not necessarily divisible. In fact, let $L$ be a field and set $R=L[x, y]$. Set $M=R /(x, y)$. Then for any flat $R$-module $N$, we have $\operatorname{Ext}_{R}^{1}(M, N)=0$, but $\operatorname{Ext}_{R}^{2}(M, R) \cong \operatorname{Hom}_{R}(M, M) \neq 0$. Hence $M$ is not torsion-free. By [7, Proposition 3.7] and [4, Lemma 3.4], $M^{+}$is copure injective. $B y$ [6, Proposition 5.3.7] and [11, Lemma $3.1 \&$ Theorem 3.3], $M^{+}$is not divisible.

Lemma 2.3. Let $R$ be a domain. Then ci $D(R) \leq 1$ if and only if every $h$-divisible module is copure injective.

Proof. The assertion follows from the fact that $\operatorname{pd}_{R} E \leq 1$ holds for any injective $R$-module $E$ by [7].

Let $M$ be an $R$-module. As in [7], the copure projective dimension $c p d_{R}(M)$ of an $R$-module $M$ is defined to be the smallest integer $n \geq 0$ such that $\operatorname{Ext}_{R}^{n+i}(M, F)=0$ 
for any flat $R$-module $F$ and for any $i \geq 0$. Of course, if no such $n$ exists, write $\operatorname{cpd}_{R}(M)=\infty$. Thus $\operatorname{cpd}(M) \leq m$ is equivalent to $M$ has a strongly copure projective resolution $0 \longrightarrow P_{m} \longrightarrow P_{m-1} \longrightarrow \cdots \longrightarrow P_{1} \longrightarrow P_{0} \longrightarrow M \longrightarrow 0$, where each $P_{i}$ is strongly copure projective. The copure projective dimension of a ring $R$ is defined as $c p D(R)=\sup \left\{c p d_{R}(M) \mid M\right.$ is an $R$-module $\}$.

We are now in a position to give an affirmative answer to Question 1.1.

Theorem 2.4. Let $R$ be a domain. Then the following statements are equivalent:

(1) $R$ is a Gorenstein Dedekind domain.

(2) $c i D(R) \leq 1$.

(3) Every divisible module is copure injective.

(4) Every h-divisible module is copure injective.

(5) Every divisible module is Gorenstein injective.

(6) Every h-divisible module is Gorenstein injective.

Proof. (1) $\Rightarrow(2)$ Let $E$ be an injective module. Then $\operatorname{pd}_{R} E \leq 1$ by [2, Theorem 1.2]. Let $X$ be any $R$-module. Then $\operatorname{Ext}_{R}^{2}(E, X)=0$ and $\operatorname{cid}_{R} X \leq 1$. Hence $c i D(R) \leq 1$.

$(2) \Rightarrow(1)$ Let $P$ be a nonzero prime ideal of $R$. Pick $0 \neq a \in P$. Set $m=$ $\operatorname{ciD}(T=R / a R)$. There is a $T$-module $\bar{M}=M / a M \neq 0$ with $\operatorname{cid}_{T} \bar{M}=m$, and an injective $T$-module $N$ with $\operatorname{Ext}_{T}^{m}(N, M) \neq 0$. Let $0 \rightarrow N \rightarrow E \rightarrow C \rightarrow 0$ be an exact sequence, where $E$ is an injective $R$-module, and $M$ is an $R$-module. Thus $C$ is also an injective $T$-module. Hence we have the exact sequence $\operatorname{Ext}_{T}^{m}(E, \bar{M}) \rightarrow$ $\operatorname{Ext}_{T}^{m}(N, \bar{M}) \rightarrow \operatorname{Ext}_{T}^{m+1}(C, \bar{M})=0$, which implies $\operatorname{Ext}_{T}^{m}(E, \bar{M}) \neq 0$. By Rees Theorem, we get $\operatorname{Ext}_{R}^{m+1}(E, M) \cong \operatorname{Ext}_{T}^{m}(E, \bar{M}) \neq 0$. Therefore, $1 \geq \operatorname{cid}_{R} M \geq$ $m+1$. Hence $m=0$. Therefore, $\operatorname{ci} D(T)=0$. Then $T$ is a $\mathrm{QF}$ ring. Since a $\mathrm{QF}$ ring is Artinian, $P /(a)$ is finitely generated. Consequently, $P$ is finitely generated, and hence $R$ is Noetherian. Thus $c p D(R) \leq 1$ by [7, Corollary 5.6]. Hence $R$ is a Gorenstein Dedekind domain by [7, Theorem 4.18].

(2) $\Leftrightarrow(3) \Leftrightarrow(4)$ By Lemma 2.1 and Lemma 2.3.

$(1) \Leftrightarrow(5) \Leftrightarrow(6)$ Since all Gorenstein Dedekind domains are Matlis domains, the result holds.

Corollary 2.5. Let $R$ be a Gorenstein Dedekind domain. Then $R$ is a Dedekind domain if and only if every copure injective $R$-module is injective.

We conclude this article with the following examples.

Rings $R$ with $c i D(R) \leq 1$ are not necessarily Noetherian. 
Example 2.6. Let $R$ be an umbrella ring with $\operatorname{gl} \cdot \operatorname{dim}(R) \leq 2$ and let $P$ be the maximum non-finitely generated prime ideal of $R$. Pick $0 \neq a \in P$. Then $R /(a)$ is a coherent ring with $c i D(R) \leq 1$, and not Noetherian.

Rings $R$ with $c i D(R) \leq 1$ are not necessarily hereditary.

Example 2.7. Construct $R=\mathbb{Q}[x, y] /\left(x^{2}+2 y^{2}\right)$. Since $x^{2}+2 y^{2}$ is an irreducible polynomial, we have that $R$ is a Gorenstein Dedekind domain. Noting that $R$ is not integrally closed, we have gl.dim $(R)=\infty$.

Let $R$ be a ring with $\operatorname{ci}(R) \leq 1$. Then $\operatorname{gl} \cdot \operatorname{dim}(R)<\infty$ is not necessarily true.

Example 2.8. We give another example of a ring with ci $D(R) \leq 1$ and $\operatorname{gl} \cdot \operatorname{dim}(R)=$ $\infty$. Set $R=\mathbb{Z}_{4}$, where $\mathbb{Z}$ is the set of integers. Then $R$ is a $\mathrm{QF}$ ring with gl.dim $(R)=\infty$.

Let $R$ be a ring with gl.dim $(R)<\infty$. Then $\operatorname{ci} D(R) \leq 1$ is not necessarily true.

Example 2.9. Let $\mathbb{C}$ be the field of complex numbers and $X, Y$ be the indeterminates over $\mathbb{C}$. We use $\mathbb{C}(X, Y)$ to denote the quotient field of the polynomial ring $\mathbb{C}[X, Y]$. Let $Z$ be an indeterminate over $\mathbb{C}(X, Y)$. Then $\mathfrak{m}=(Z)$ is a maximal ideal of $\mathbb{C}(X, Y)$. Construct $R=\mathbb{C}[X, Y]+Z \mathbb{C}(X, Y)[Z]_{\mathfrak{m}}$. Then gl.dim $(R)=3$ and $\operatorname{ci} D(R)>1$.

Let $R$ be a ring with $\operatorname{gl} \cdot \operatorname{dim}(R)=\infty$. Then $\operatorname{ci} D(R) \leq 1$ does not necessarily hold.

Example 2.10. Construct a ring $R=\mathbb{Z}_{4}[X, Y]$, where $X, Y$ are the indeterminates over $\mathbb{Z}_{4}$. Then $\operatorname{gl} \cdot \operatorname{dim}(R)=\infty$ and $\operatorname{ci} D(R)>1$.

Acknowledgment. The author would like to thank the referee for the valuable suggestion and comments.

\section{References}

[1] S. Bazzoni and L. Salce, Almost perfect domains, Colloq. Math., 95(2) (2003), 285-301.

[2] D. Bennis, A short survey on Gorenstein global dimension, Actes des rencontres du C.I.R.M., 2(2) (2010), 115-117.

[3] D. Bennis and N. Mahdou, Global Gorenstein dimensions, Proc. Amer. Math. Soc., 138(2) (2010), 461-465.

[4] E. E. Enochs and O. M. G. Jenda, Copure injective resolutions, flat resolvents and dimensions, Comment. Math. Univ. Carolin., 34(2) (1993), 203-211. 
[5] E. E. Enochs and O. M. G. Jenda, Gorenstein injective and projective modules, Math. Z., 220(4) (1995), 611-633.

[6] E. E. Enochs and O. M. G. Jenda, Relative Homological Algebra, de Gruyter Exp. Math., Vol. 30, Walter de Gruyter, Berlin, 2000.

[7] X. H. Fu, H. Y. Zhu and N. Q. Ding, On copure projective modules and copure projective dimensions, Comm. Algebra, 40(1) (2012), 343-359.

[8] L. Fuchs and S. B. Lee, Weak-injectivity and almost perfect domains, J. Algebra, 321(1) (2009), 18-27.

[9] R. Hamsher, On the structure of a one dimensional quotient field, J. Algebra, 19 (1971), 416-425.

[10] S. B. Lee, h-Divisible modules, Comm. Algebra, 31(1) (2003), 513-525.

[11] S. B. Lee, Weak-injective modules, Comm. Algebra, 34(1) (2006), 361-370.

\section{Tao Xiong}

College of Mathematics and Information

China West Normal University

637002 Nanchong, P. R. China

e-mail: Taoxiong2004@163.com 